\title{
Variability and the X-ray/UV ratio of active galactic nuclei ${ }^{\star}$
}

\section{Analysis of a low-redshift Swift sample}

\author{
F. Vagnetti ${ }^{1,2}$, M. Antonucci ${ }^{1}$, and D. Trevese ${ }^{2}$ \\ 1 Dipartimento di Fisica, Università di Roma "Tor Vergata”, via della Ricerca Scientifica 1, 00133 Roma, Italy \\ e-mail: fausto.vagnetti@roma2.infn.it \\ 2 Dipartimento di Fisica, Università di Roma "La Sapienza", Piazzale Aldo Moro 2, 00185 Roma, Italy
}

Received 25 September 2012 / Accepted 14 December 2012

\section{ABSTRACT}

\begin{abstract}
Context. Variability, both in X-ray and optical/UV, affects the well-known anti-correlation between the $\alpha_{\mathrm{ox}}$ spectral index and the UV luminosity of active galactic nuclei, contributing part of the dispersion around the average correlation (intra-source dispersion) in addition to the differences among the time-average $\alpha_{\mathrm{ox}}$ values from source to source (inter-source dispersion).

Aims. We aim to evaluate the intrinsic $\alpha_{\mathrm{ox}}$ variations in individual objects and their effect on the dispersion of the $\alpha_{\mathrm{ox}}-L_{\mathrm{UV}}$ anticorrelation.

Methods. We used simultaneous UV/X-ray data from Swift observations of a low-redshift sample to derive the epoch-dependent $\alpha_{\text {ox }}(t)$ indices. We corrected for the host galaxy contribution by a spectral fit of the optical/UV data. We computed ensemble structure functions to analyse the variability of multi-epoch data.

Results. We find a strong intrinsic $\alpha_{\mathrm{ox}}$ variability, which significantly contributes ( $40 \%$ of the total variance) to the dispersion of the $\alpha_{\mathrm{ox}}-L_{\mathrm{UV}}$ anti-correlation (intra-source dispersion). The strong X-ray variability and weaker UV variability of this sample are comparable to other samples of low- $z$ active galactic nuclei, and are neither caused by the high fraction of strongly variable narrow line Seyfert 1 galaxies, nor by dilution of the optical variability by the host galaxies. Dilution instead affects the slope of the anticorrelation, which steepens, once corrected, and becomes similar to higher luminosity sources. The structure function of $\alpha_{\mathrm{ox}}$ increases with the time lag up to about one month. This indicates the important contribution of the intermediate-to-long timescale variations, which are possibly generated in the outer parts of the accretion disk.
\end{abstract}

Key words. surveys - galaxies: active - quasars: general - X-rays: galaxies

\section{Introduction}

The X-ray-to-UV ratio of active galactic nuclei (AGN) gives direct information on an important region of the spectral energy distribution (SED), relating the radiative processes that operate in the accretion disk and in the corona, connecting their emissions across the unobservable band of the extreme UV. It characterises the shape of the SED, and affects, through the ionisation equilibrium, properties of the UV spectral lines, such as the equivalent width and the blue-shift of the CIV $\lambda 1549$ emission line (Richards et al. 2011).

The X-ray/UV ratio is often expressed through the inter-band spectral index

$\alpha_{\mathrm{ox}} \equiv \frac{\log \left(L_{X} / L_{\mathrm{UV}}\right)}{\log \left(v_{X} / \nu_{\mathrm{UV}}\right)}=0.384 \log \left(\frac{L_{X}}{L_{\mathrm{UV}}}\right)$

between the conventional frequencies $v_{X} \equiv v_{2 \mathrm{keV}}$ and $v_{\mathrm{UV}} \equiv$ $v_{2500 \AA}$.

$\alpha_{\text {ox }}$ is found to be strongly anti-correlated with the ultraviolet specific luminosity $L_{\mathrm{UV}}$, showing that more luminous objects are, on average, relatively weaker in X-rays:

$\alpha_{\mathrm{ox}}=a \log L_{\mathrm{UV}}+$ const $\quad(a<0)$.

This relation has been studied by many authors, who found slopes approximately in the interval $-0.2 \lesssim a \lesssim-0.1$ (e.g.,

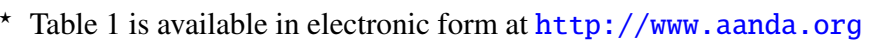

Strateva et al. 2005; Steffen et al. 2006; Just et al. 2007; Gibson et al. 2008; Grupe et al. 2010; Vagnetti et al. 2010), depending on the selection of the sample, and especially on its range of luminosities and/or redshifts. For instance, while Just et al. (2007) derived $a=-0.14$ within a wide area of the $L-z$ plane, $27.5<\log L_{\mathrm{UV}}<33,0<z<6$, a flatter slope was found by Grupe et al. (2010), $a=-0.114$, for a low-luminosity and low-redshift sample, $26<\log L_{\mathrm{UV}}<31, z<0.35$, and a steeper slope, $a=-0.217$, was obtained by Gibson et al. (2008) for higher redshifts and luminosities, $30.2<\log L_{\mathrm{UV}}<31.8$, $1.7<z<2.7$. A similar trend was found dividing a wider sample into two subsamples with lower and higher luminosity or redshift, see e.g. Steffen et al. (2006); Vagnetti et al. (2010). Thus, a precise estimate of the slope cannot be given in general terms, as the $\alpha_{\mathrm{ox}}-L_{\mathrm{UV}}$ relation itself might be non-linear.

Moreover, Gibson et al. (2008) noticed the large scatter of the data around the average relation, suggesting that a large part of it can be caused by variability, combined with nonsimultaneity of the X-ray and optical observations. In a previous paper (Vagnetti et al. 2010, Paper I), we have analysed a sample with simultaneous measurements extracted from the XMM-Newton Serendipitous Source Catalogue (Watson et al. 2009), concluding that artificial $\alpha_{\text {ox }}$ variability due to nonsimultaneity is not the main cause of dispersion, while intrinsic $\alpha_{\text {ox }}$ variability of individual sources (or intra-source dispersion) and intrinsic differences in the time-average values of $\alpha_{\text {ox }}$ from source to source (or inter-source dispersion) are the most important contributions. 
In Paper I, we then analysed the $\alpha_{\mathrm{ox}}$ variability computing the ensemble structure function, and pointed out the need of more AGN samples with simultaneous measurements to make progress in this topic. Appropriate data can be obtained by space observatories that have both X-ray and optical/UV telescopes on board, such as XMM-Newton and Swift.

In this paper, we present the analysis of a sample of lowredshift AGNs observed by Swift that were previously studied by Grupe et al. (2010), whose paper and sample will hereafter be referred to as G10.

This paper is organised as follows. Section 2 describes the data extracted from G10 and from the Swift archive. Section 3 analyses the $\alpha_{\mathrm{ox}}-L_{\mathrm{UV}}$ anti-correlation and its dispersion. In Sect. 4, we present the ensemble structure function of the intrinsic X/UV variability. Section 5 discusses and summarises the results.

Throughout the paper, we adopt the cosmology $H_{0}=$ $70 \mathrm{~km} \mathrm{~s}^{-1} \mathrm{Mpc}^{-1}, \Omega_{\mathrm{m}}=0.3$, and $\Omega_{\Lambda}=0.7$.

\section{Data}

The G10 sample consists of 92 AGNs extracted from the bright soft X-ray selected sample of Grupe et al. (2001), which was observed with Swift between 2005 and 2010. The sample by Grupe et al. (2001) contains all 110 Seyfert galaxies from the sample of 397 sources by Thomas et al. (1998), which was extracted from the ROSAT All Sky Survey (Voges et al. 1999) to include sources selected to be X-ray bright (count rate $>0.5$ counts $/ \mathrm{s}$ ), $\mathrm{X}$-ray soft (hardness ratio ${ }^{1} \mathrm{HR}<0.0$ ), and at high Galactic latitude $\left(|b|>20^{\circ}\right)$.

The G10 sample includes simultaneous X-ray and opti$\mathrm{cal} / \mathrm{UV}$ measurements for most of the sources, and in many cases multi-epoch observations are available, with a total of 299 observations for the 92 sources. However, in a few cases the data are not usable for our purposes, because of lack of X-ray or optical/UV measurements. We therefore adopted a preliminary subsample of 90 sources, with 74 multi-epoch sources and 16 single-epoch sources, for a total of 241 observations. In the following analysis (Sect. 2.1), we removed a few observations whose determination of the AGN luminosity is unreliable, because of strong dominance of the host galaxy, or because of insufficient spectral coverage of the optical/UV data. We define the resulting set of 216 observations as sample A, including 86 sources (68 multi-epoch and 18 single-epoch). In Sect. 3, we introduce another subsample that does not contain known radioloud sources, which we call sample B. The data are taken from the tables of the G10 paper, which are available electronically, and were checked through the Swift archive at Heasarc ${ }^{2}$.

Compared to the sample of Paper I, the sample studied in the present paper lies in a region of the luminosity-redshift plane at lower redshifts $(z<0.35)$ and luminosities $\left(26<\log L_{\mathrm{UV}}<31\right)$, see Fig. 1. The relevant properties of the sources of samples A and $\mathrm{B}$ at each epoch are reported in Table 1, where Col. 1 corresponds to the source serial number according to G10; Col. 2 to the source name; Col. 3 to the observation epoch serial number; Col. 4 to the epoch in modified Julian days (MJD); Col. 5 to the redshift; Col. 6 to the soft X-ray spectral index, according to Table 4 of G10; Col. 7 to the logarithm of the specific luminosity at $2 \mathrm{keV}$ in erg s${ }^{-1} \mathrm{~Hz}^{-1}$; Col. 8 to the logarithm of the specific AGN luminosity at $2500 \AA$ in erg s${ }^{-1} \mathrm{~Hz}^{-1}$; Col. 9 to the logarithm of the specific host galaxy luminosity at $2500 \AA$

\footnotetext{
1 With reference to the ROSAT bands $0.1-0.4 \mathrm{keV}$ and $0.5-2.4 \mathrm{keV}$.

2 http://heasarc.gsfc.nasa.gov/
}

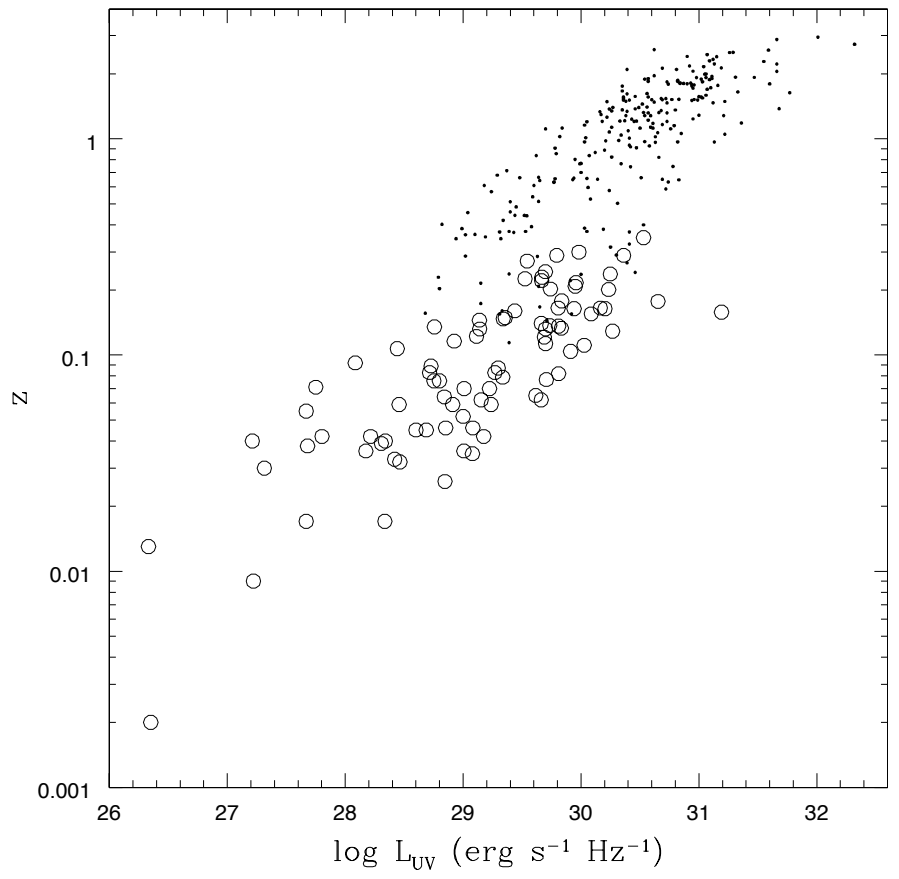

Fig. 1. Distribution of the sources in the $L_{\mathrm{UV}}-z$ plane. Open circles: Swift sample; dots: XMM-Newton sample.

in $\mathrm{erg} \mathrm{s}^{-1} \mathrm{~Hz}^{-1}$ (substituted by a hyphen when the galaxy contribution is negligible); Col. 10 to the optical/X-ray spectral index; and Col. 11 to the radio-loudness flag $f_{\mathrm{RL}}=1$ (radio-loud), $f_{\mathrm{RL}}=0$ (radio-quiet), $f_{\mathrm{RL}}=-1$ (unclassified).

\subsection{UV luminosities}

The UltraViolet/Optical Telescope (UVOT) onboard Swift has six photometric filters, whose central wavelengths are $\lambda(V)=$ $5468 \AA, \lambda(B)=4392 \AA, \lambda(U)=3465 \AA, \lambda(U V W 1)=2600 \AA$, $\lambda(U V M 2)=2246 \AA$, and $\lambda(U V W 2)=1928 \AA$ (Roming et al. $2005,2009)$. Magnitudes in one or more of these bands are available for each source and epoch of our sample from Table 3 of G10. We first transformed magnitudes to fluxes according to the formulae given by Poole et al. (2008), using the count rate-to-flux conversion factors of their table 10 (GRB models, also appropriate for AGNs). To estimate UV luminosities at $2500 \AA$, similarly to the procedure used in Paper I, we computed, from each of the available fluxes, the corresponding luminosities as $L_{v}\left(v_{\text {rest }}\right)=F_{v}\left(v_{\text {obs }}\right) 4 \pi D_{\mathrm{L}}^{2} /(1+z)$, and derived the rest-frame SEDs, which are shown in Fig. 2.

Then, we took into account the contribution of the host galaxy starlight, which can be significant for AGNs of low luminosity such as those considered here. Following a procedure similar to that adopted by Lusso et al. (2010), we modelled the optical spectrum by a combination of AGN and galaxy components as

$L_{v}=A\left[f_{\mathrm{A}} F_{\mathrm{R}}(v)+f_{\mathrm{G}}\left(v / v_{*}\right)^{-3}\right]$,

where $F_{\mathrm{R}}(v)$ is the mean SED computed by Richards et al. (2006) for type 1 quasars from the Sloan Digital Sky Survey (SDSS), $A$ is a normalisation factor, and the coefficients $f_{\mathrm{G}}$ and $f_{\mathrm{A}}$ represent the galaxy and $\mathrm{AGN}$ fractional contributions at the frequency $v_{*}$, corresponding to $2500 \AA\left(\log v_{*}=15.08\right)$. The average spectral index $\alpha_{\text {opt }}$ of Eq. (3) is a monotonic function of the ratio $f_{\mathrm{G}} / f_{\mathrm{A}}$, which is thus determined by comparison 
F. Vagnetti et al.: X-ray/UV ratio of AGNs. II.

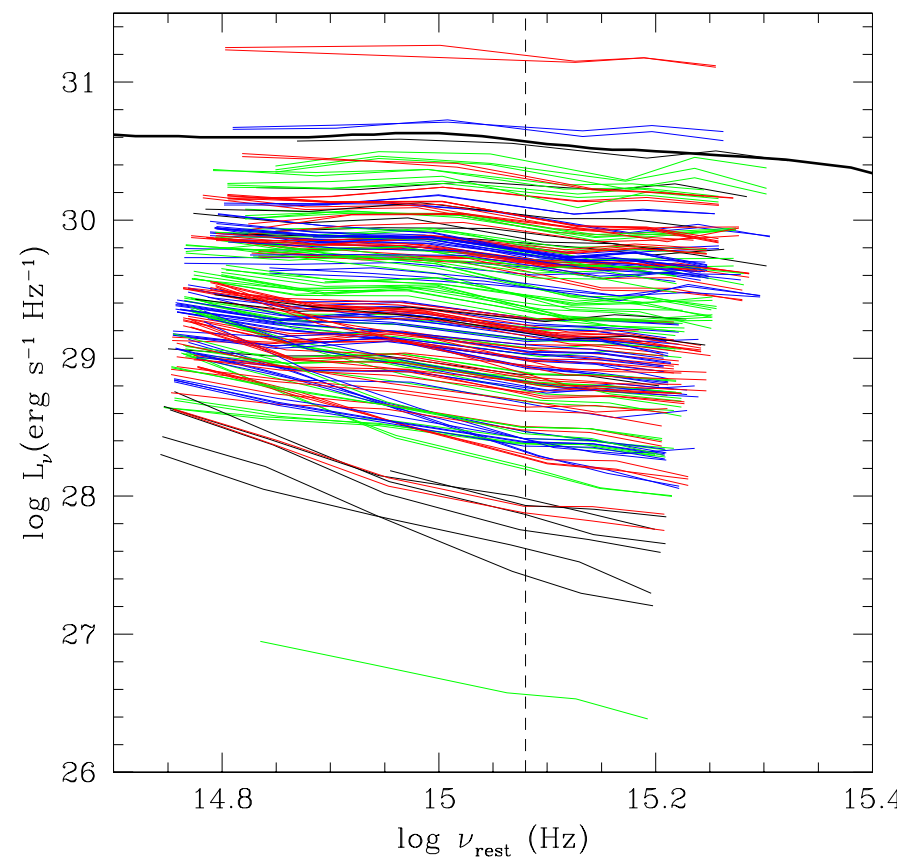

Fig. 2. Spectral energy distributions from the available UVOT data. Black lines refer to sources with data at a single epoch, while coloured lines refer to multi-epoch sources. Data from the same source are plotted with the same colour, but more sources are represented with the same colour. The continuous curve that covers the whole range of the plot is the average SED computed by Richards et al. (2006) for type 1 quasars from the SDSS.

with the slope of each observed SED. A clear sign of this dependence is apparent in Fig. 2, where less luminous sources have progressively steeper spectra.

The normalisation factor $A$ is then fitted to the data by general linear least-squares (Press et al. 1992) as

$A=\frac{\sum_{i=1}^{N} y_{i} X\left(v_{i}\right) / \sigma_{i}^{2}}{\sum_{i=1}^{N}\left[X\left(v_{i}\right)\right]^{2} / \sigma_{i}^{2}}$,

where $X\left(v_{i}\right)=\log L_{v}\left(v_{i}\right)$ is given by the model function of Eq. (3) computed in correspondence to the available UVOT rest-frame frequencies $v_{i}, y_{i}=\log L_{i}$ is given by the corresponding measured specific luminosities, and $\sigma_{i}$ are their errors. This procedure determines the luminosities of the two components at $2500 \AA, L_{\mathrm{AGN}}$ and $L_{\mathrm{G}}$, for each source and epoch. In most cases, we can compare the different determinations of $L_{\mathrm{G}}$ for the same source at more epochs, finding low dispersions (usually $\lesssim 0.15$ in $\log L_{\mathrm{G}}$ ). We then fixed $L_{\mathrm{G}}$ to its average value for each source and repeated the fit to the data, modifying the fitting function as

$L_{v}=A^{\prime} F_{\mathrm{R}}(v)+L_{\mathrm{G}}\left(v / v_{*}\right)^{-3}$,

where the factor $A^{\prime}$ is now given by

$A^{\prime}=\frac{\sum_{i=1}^{N} y_{i}^{\prime} X^{\prime}\left(v_{i}\right) / \sigma_{i}^{2}}{\sum_{i=1}^{N}\left[X^{\prime}\left(v_{i}\right)\right]^{2} / \sigma_{i}^{2}}$,

with $X^{\prime}\left(v_{i}\right)=\log F_{\mathrm{R}}\left(v_{i}\right)$, and $y_{i}^{\prime}=\log \left[L_{i}-L_{\mathrm{G}}\left(v_{i} / v_{*}\right)^{-3}\right]$. The AGN luminosity at $2500 \AA$ is then given by $L_{\mathrm{AGN}}=A^{\prime} F_{\mathrm{R}}\left(v_{*}\right)$. In the following, we refer to it simply as $L_{\mathrm{UV}}$, maintaining the name $L_{\mathrm{G}}$ for the galactic contribution.
For a subsample of ten sources, Hubble Space Telescope observations by Bentz et al. (2009) are available, with direct measurements of the AGN and galactic luminosities. Our estimated values of $L_{\mathrm{G}}$ are consistent with these measurements.

In some cases, when the number of available UVOT data is $<4$, only a small portion of the SED is sampled, and the two contributions cannot be determined. This occurs for 19 observations in total, leading to the removal of two sources from our sample and to the decrease of the number of useful observations for some of the remaining sources. We also removed two more sources for which the slope of the observed SED is steeper than -3 , indicating a negligible AGN contribution. This means that we removed four sources in total, defining our sample A, which includes 86 sources (68 multi-epoch and 18 singleepoch), to have a total of 216 observations. The galactic dilution is substantial $\left(f_{\mathrm{G}} \gtrsim 30 \%\right.$ ) for 15 sources out of the 86 sources of sample A. This is discussed in more detail in Sect. 3.3.

\subsection{X-ray luminosities}

Unabsorbed rest-frame soft-band X-ray fluxes, $F_{\mathrm{X}}(0.2-2 \mathrm{keV})$, are given by G10, together with the soft-X-ray spectral index $\alpha_{x}$ (defined according to the rule $F_{v} \propto v^{-\alpha_{x}}$ ). We derived the specific flux at $2 \mathrm{keV}$ as

$F_{v}(2 \mathrm{keV})=\frac{F_{X}(0.2-2 \mathrm{keV})}{v_{2 \mathrm{keV}}} f\left(\alpha_{x}\right)$,

with $f\left(\alpha_{x}\right)=\left(\alpha_{x}-1\right) /\left(10^{\alpha_{x}-1}-1\right)$ for $\left(\alpha_{x} \neq 1\right)$ and $f\left(\alpha_{x}\right)=$ $1 / \ln 10$ for $\left(\alpha_{x}=1\right)$, and computed the specific luminosities accordingly.

\section{3. $\alpha_{\mathrm{ox}}-L_{\mathrm{UV}}$ anti-correlation}

Radio-loud (RL) quasars are known to be relatively X-ray bright because of the enhanced X-ray emission associated with their jets (e.g. Zamorani et al. 1981; Worrall et al. 1987); in contrast, broad absorption line (BAL) quasars are relatively X-ray faint, compared to non-BAL quasars (e.g. Green \& Mathur 1996; Brandt et al. 2000; Gibson et al. 2008). Both populations are therefore usually removed from the analysis of the $\alpha_{\mathrm{ox}}-L_{\mathrm{UV}}$ anti-correlation (e.g. Just et al. 2007; Gibson et al. 2008; Young et al. 2010; Vagnetti et al. 2010).

We found radio information from the NASA/IPAC Extragalactic Database (NED) for 35 sources out of 86; we used the data at $5 \mathrm{GHz}$ when available, or scaled the flux as $f_{v} \propto$ $v^{-0.8}$ when observations were available at a different frequency. We classified the sources as RL when the inequality $R^{*}=$ $L_{v}(5 \mathrm{GHz}) / L_{\mathrm{UV}}>10$ was satisfied (e.g., Sramek \& Weedman 1980; Kellermann et al. 1989), marking them with $f_{\mathrm{RL}}=1$ in Table 1 (9 sources out of 35 ). Sources with $R^{*}<10$ were classified as radio-quiet (RQ) and marked with $f_{\mathrm{RL}}=0$ (26 out of 35). Sources without radio information (51 out of 86 ) were marked with $f_{\mathrm{RL}}=-1$. Concerning the presence of BAL quasars among our sources, we checked several studies about low-redshift BALs (Pettini \& Boksenberg 1985; Turnshek \& Grillmair 1986; Kinney et al. 1991; Turnshek et al. 1997; Sulentic et al. 2006; Ganguly et al. 2007) but found no coincidences. Although both radio and BAL information are quite incomplete, we finally removed only 9 RL AGNs from sample A, which then defines a reference sample of 77 sources (61 multi-epoch +16 single epoch, sample B) for our subsequent analysis. Sample B includes 194 observations listed in Table 1 with $f_{\mathrm{RL}} \neq 1$. 


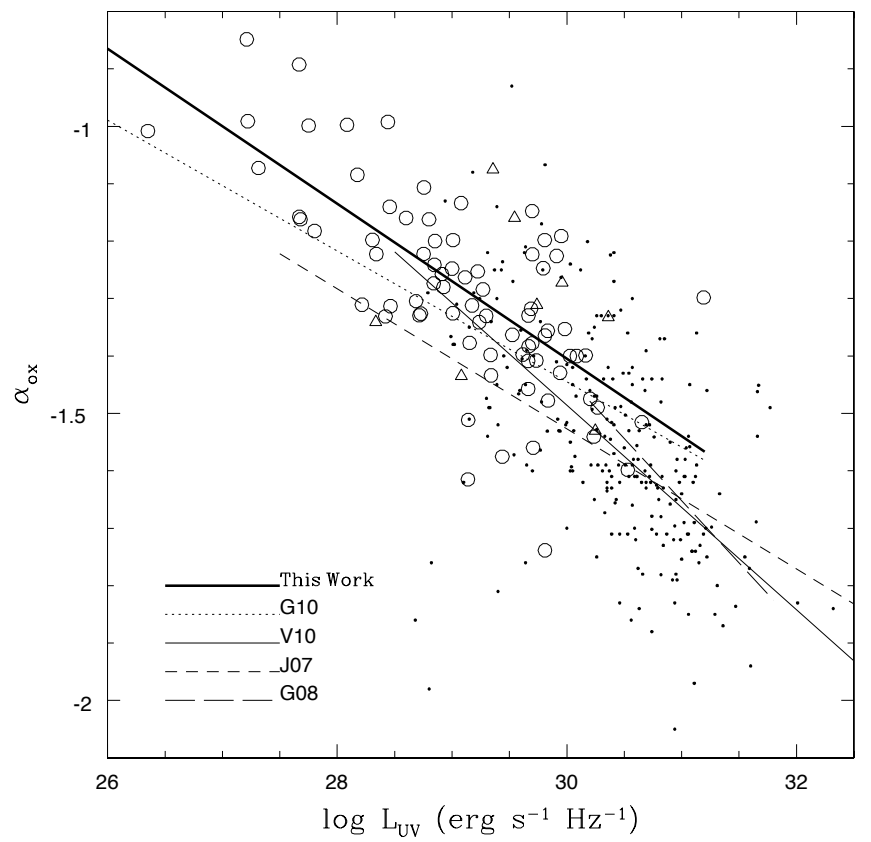

Fig. 3. $\alpha_{\mathrm{ox}}$ as a function of the $2500 \AA$ specific luminosity $L_{\mathrm{UV}}$, for samples A (circles and triangles) and B (circles) and for the sample of Paper I (dots). Triangles refer to radio-loud sources, circles to radio-quiet and radio-unclassified sources. Linear fits are shown for the present work, and are marked for previous works G10 (Grupe et al. 2010), V10 (Paper I), J07 (Just et al. 2007), G08 (Gibson et al. 2008).

Figure 3 shows the distribution of sources (sample A, circles and triangles; sample $\mathrm{B}$, circles) in the plane $\alpha_{\mathrm{ox}}-L_{\mathrm{UV}}$, compared with the XMM-Newton sample (dots) studied in Paper I. The average values of $\alpha_{\mathrm{ox}}$ and $L_{\mathrm{UV}}$ are shown for multi-epoch sources. Also shown is the linear least-squares fit for the Swift sample B:

$\alpha_{\mathrm{ox}}=(-0.135 \pm 0.015) \log L_{\mathrm{UV}}+(2.645 \pm 0.446)$

(thick continuous line). Moreover, the fit for the XMM-Newton reference sample of Paper I, $\alpha_{\mathrm{Ox}}=(-0.178 \pm 0.014) \log L_{\mathrm{UV}}+$ $(3.854 \pm 0.420)$ (thin continuous line), and the fit obtained by G10, $\alpha_{\mathrm{ox}}=(-0.114 \pm 0.014) \log L_{\mathrm{UV}}+(1.975 \pm 0.403)$ (with luminosities scaled to cgs units, dotted line) are shown. Our present fit is somewhat steeper than that of G10 because we corrected for the galactic dilution. Both fits are much flatter than our fit of Paper I, which was derived from higher luminosity sources. For an additional comparison, the fits by Just et al. (2007), $\alpha_{\mathrm{ox}}=(-0.140 \pm 0.007) \log L_{\mathrm{UV}}+(2.705 \pm 0.212)$, and by Gibson et al. (2008), $\alpha_{\mathrm{ox}}=(-0.217 \pm 0.036) \log L_{\mathrm{UV}}+(5.075 \pm$ 1.118) are shown. There is a clear tendency for a steepening of the $\alpha_{\mathrm{ox}}-L_{\mathrm{UV}}$ anti-correlation for samples extending at higher luminosities, as already mentioned in the introduction and discussed in previous works (Steffen et al. 2006; Vagnetti et al. 2010).

\subsection{Dispersion}

We define the residuals

$\Delta \alpha_{\mathrm{ox}}=\alpha_{\mathrm{ox}}-\alpha_{\mathrm{ox}}\left(L_{\mathrm{UV}}\right)$,

adopting Eq. (8) as our reference $\alpha_{\mathrm{ox}}\left(L_{\mathrm{UV}}\right)$ relation. The standard deviation of our distribution of the residuals is $\sigma=0.124$ for sample A and $\sigma=0.117$ for sample B. The dispersion in our $\Delta \alpha_{\text {ox }}$ distribution is of the same order as those obtained in some studies based on non-simultaneous X-ray and UV data.

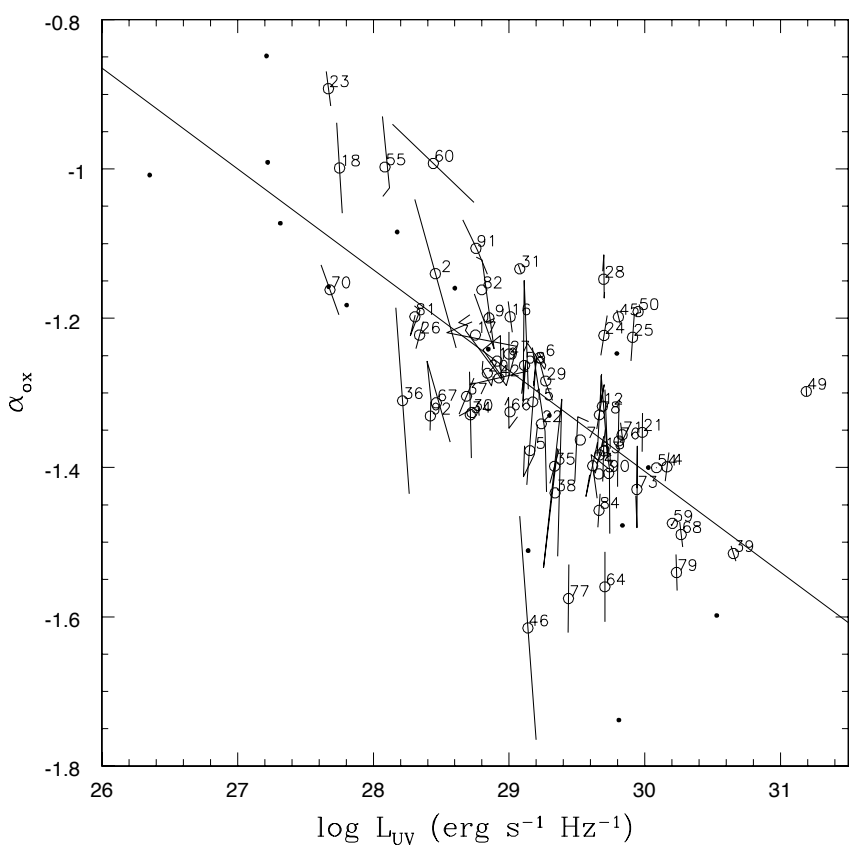

Fig. 4. Tracks of individual sources of sample B in the plane $\alpha_{\mathrm{ox}}-L_{\mathrm{UV}}$. Connected segments show the tracks of multi-epoch sources, while open circles represent the average values of the same sources, which are labelled with their serial numbers as in Table 1. Objects with singleepoch measurements are represented by dots. The straight line is the adopted $\alpha_{\mathrm{ox}}-L_{\mathrm{UV}}$ relation, Eq. (8).

Indeed, it is lower than those found by Strateva et al. (2005, e.g.) (0.14) and by Young et al. (2010) (0.16), but slightly higher than that evaluated by Gibson et al. (2008) (0.10). Values found in previous simultaneous studies are also of the same order, e.g. our XMM-Newton sample of Paper I (0.12), and the small clean catalogue by Wu et al. (2012) (0.12). Hence we confirm our conclusion of Paper I, that non-simultaneity of X-ray and UV measurements, which we call artificial $\alpha_{\mathrm{ox}}$ variability, is not the main contributor to the dispersion of the residuals $\Delta \alpha_{\mathrm{ox}}$. Wu et al. (2012) reached the opposite conclusion, but they compared their results only with those of Just et al. (2007) (0.15). Nonsimultaneity would lead to an artificial change of $\alpha_{\text {ox }}$ caused by the sole change of the X-ray flux in the time elapsed from the optical measurement, or vice versa. An average change of $15-30 \%$ in a few years would apply for the optical case (see e.g. Wilhite et al. 2008; MacLeod et al. 2012), and 40-50\% for the X-ray case (Markowitz \& Edelson 2004; Vagnetti et al. 2011). Applying Eq. (1), this would translate into an $\alpha_{\text {ox }}$ artificial change of $\$ 0.07$. On the other hand, as we have shown in Paper I, and as we will discuss in more detail below, there is a sizable intrinsic $\alpha_{\text {ox }}$ variability that we estimate to be $\sim 0.07$, large enough to provide a significant contribution, at least of the same order as the artificial variability, even when the latter is removed by simultaneous X/UV measurements.

\subsection{Tracks of individual sources}

Multi-epoch information is available for 68/86 sources of sample A and for 61/77 sources of sample B. We show in Fig. 4 the tracks of individual sources in the $\alpha_{\mathrm{ox}}-L_{\mathrm{UV}}$ plane for sample B. Strong variations in $\alpha_{\text {ox }}$ are clearly occurring for many sources, and most tracks appear to be almost vertical, suggesting the occurrence of strong changes in X-rays, and/or weak changes in $L_{\mathrm{UV}}$. This is confirmed by the histograms of the individual variability dispersions of $\log L_{\mathrm{UV}}$ and $\log L_{\mathrm{X}}$ for multi-epoch 


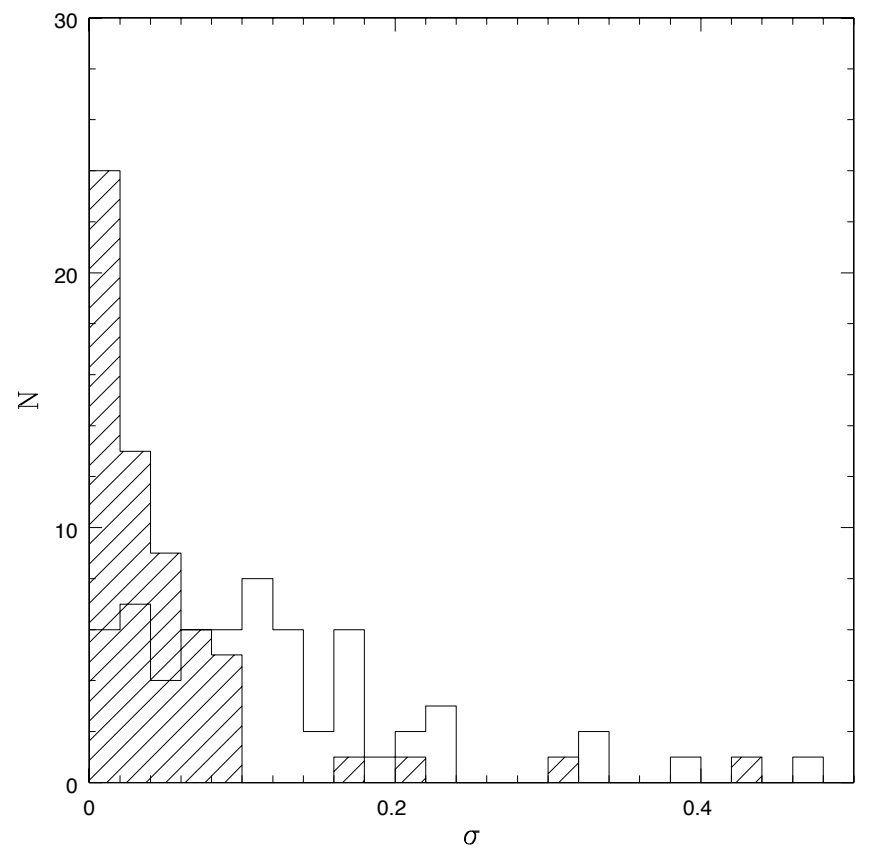

Fig. 5. Histograms of the individual variability dispersions of $\log L_{\mathrm{UV}}$ and $\log L_{X}$ for multi-epoch sources: shaded histogram, UV; empty histogram, X-ray.

sources, shown in Fig. 5. The variations occur on various time scales from days to years, therefore a better evaluation of the variability properties will be made in the next section. We note, however, that some factors could affect this apparent behaviour, e.g. the presence of a large number of narrow line Seyfert 1 (NLS1) nuclei, which are known to have strong X-ray variability (e.g. Leighly 1999) and moderate optical variability (e.g. Ai et al. 2010). We instead corrected for the effect of the other important factor, dilution of the optical variability by the host galaxy.

\subsection{Effect of the host galaxy}

Although we have subtracted host galaxy luminosities in Sect. 2.1, it is useful to discuss the possible effects of their contributions, for comparison with the literature. Wilkes et al. (1994) first pointed out that contamination by host galaxy starlight could affect the $\alpha_{\mathrm{Ox}}-L_{\mathrm{UV}}$ relation, and that excluding the lowest luminosity AGNs would cause a marginal steepening of the relation. G10 mentioned the possibility that the measured magnitudes are affected by a contribution of the host galaxy starlight within the UVOT standard extraction radius of 5 arcsec, estimating this effect to be important for a few extreme cases like Mark 493. Wu et al. (2012) analysed a large sample of quasars on wide $L$ and $z$ intervals, and pointed out that their $\alpha_{\mathrm{Ox}}-L_{\mathrm{UV}}$ slope decreases from -0.16 to -0.14 when the G10 sample is added, arguing that the difference in slopes is likely caused by host galaxy contamination at low redshift. Lusso et al. (2010) modelled the optical spectrum as a combination of AGN and galaxy components, $L_{v}=A v^{-0.5}+G v^{-3}$, and estimated the galaxy contribution from the measure of the optical spectral index. This enabled the authors to correct their $\alpha_{\mathrm{ox}}-L_{\mathrm{UV}}$ relation, which results in a steepening from -0.154 to -0.197 . $\mathrm{Xu}$ (2011) analysed a sample of low-luminosity AGNs, including 28 local Seyfert galaxies and 21 low-ionization nuclear emission-line regions (LINERs), with $L_{\mathrm{UV}}$ luminosities in the range $10^{22}-10^{27.7} \mathrm{erg} \mathrm{s}^{-1} \mathrm{~Hz}^{-1}$. The author took the nuclear magnitudes directly observed by Ho \& Peng (2001) with the Hubble Space Telescope, or estimated from $\mathrm{H}_{\beta}$ luminosity, and

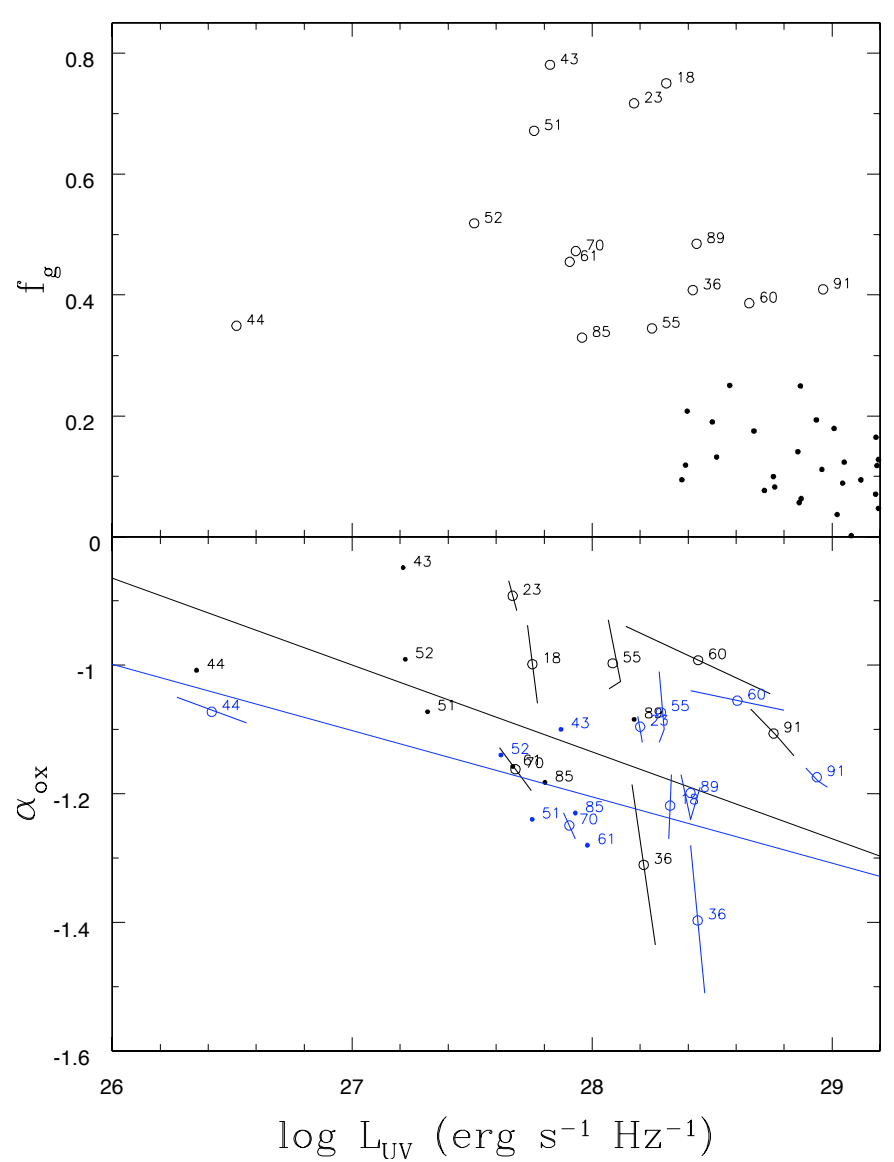

Fig. 6. Effect of the dilution by the host galaxy. Upper panel: galactic fraction $f_{\mathrm{g}}$ as a function of the UV luminosity; sources with $f_{\mathrm{g}}>30 \%$ are represented by circles and are numbered, sources with $f_{\mathrm{g}}<30 \%$ are shown as dots. Lower panel: the $\alpha_{\mathrm{ox}}-L_{\mathrm{UV}}$ relation before (blue) and after (black) correction for galaxy dilution. Only the most diluted sources are shown, all shifted along lines with slope -0.384 , by amounts increasing with $f_{\mathrm{g}}$. Sources 44 and 89 have a reduced number of epochs after correction, due to the requirement that the SED contains at least four UVOT points, as discussed in Sect. 2.1.

found for the relation $\alpha_{\mathrm{ox}}-L_{\mathrm{UV}}$ a steeper slope $(-0.134)$ than G10, but similar to our result of Eq. (8) and to that found by Just et al. (2007) for higher luminosity AGNs.

While Eq. (8) is corrected for galactic dilution, we computed the same relation for uncorrected (diluted) luminosities as well, $\alpha_{\mathrm{ox}}=(-0.103 \pm 0.016) \log L_{\mathrm{UV}}+(1.679 \pm 0.472)$, which is flatter. The dilution effect is shown in Fig. 6, where some of the low-luminosity sources are shifted towards higher $L_{\mathrm{UV}}$ and lower $\alpha_{\mathrm{ox}}$, along lines with slope -0.384 , according to Eq. (1). This slope is higher than the anti-correlation slope, especially in the low-luminosity range, and determines a flattening of the observed anti-correlation.

\section{Structure functions}

We now computed for the 59 multi-epoch sources of sample B an ensemble structure function (SF) to describe the variability of $\alpha_{\text {ox }}$ as a function of the rest-frame time lag $\tau$. We define this as in di Clemente et al. (1996), and in agreement with the procedure used in Paper I:

$S F_{\alpha}(\tau)=\sqrt{\pi / 2}\left\langle\left|\alpha_{\mathrm{ox}}(t+\tau)-\alpha_{\mathrm{ox}}(t)\right|\right\rangle$ 


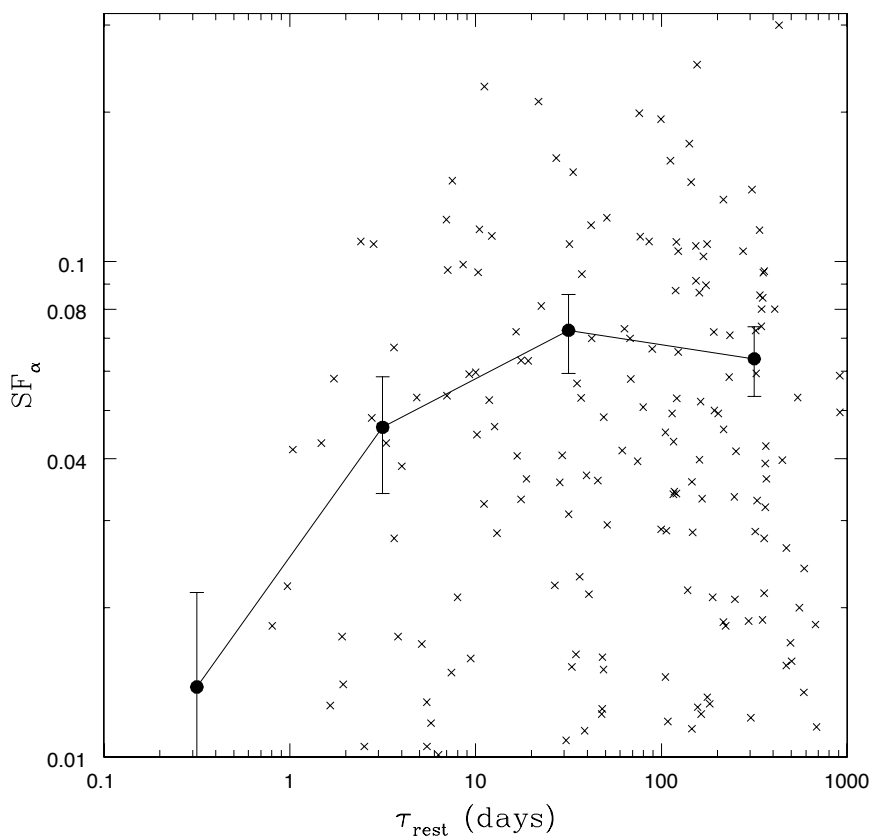

Fig. 7. Structure function of $\alpha_{\text {ox }}(t)$ vs. the rest-frame time lag for sample $B$. The crosses represent the variations of individual sources for any pair of epochs. The filled circles connected by continuous lines represent the binned ensemble structure function.

where $t$ and $t+\tau$ are two epochs, in the rest-frame, at which $\alpha_{\text {ox }}$ is determined. The factor $\sqrt{\pi / 2}$ is introduced ${ }^{3}$ to normalise the $\mathrm{SF}$ to the rms value in the case of a Gaussian distribution, and the angular brackets indicate the ensemble average over appropriate bins of time lag.

The SF, displayed in Fig. 7, shows an average increasing behaviour. Maximum variations are $\sim 0.073$ at $\sim 1$ month restframe, and can be compared with the total dispersion in the residuals, $\sigma \sim 0.117$.

As found in Paper I, variability in $\alpha_{\mathrm{ox}}$ for individual sources accounts for a large part of the observed dispersion around the average $\alpha_{\mathrm{Ox}}-L_{\mathrm{UV}}$ correlation. We call this intra-source dispersion, while the scatter of the time-average of $\alpha_{\text {ox }}$ values for individual sources constitutes the inter-source dispersion. The overall variance is then

$\sigma^{2}=\sigma_{\text {intra-source }}^{2}+\sigma_{\text {inter-source }}^{2}$

Inserting the values 0.073 and 0.117 that we obtained for the intra-source and total dispersions, into Eq. (11) indicates a $\sim 40 \%$ contribution of the intra-source dispersion to the total variance $\sigma^{2}$, similar to Paper I.

\subsection{X-ray SF}

It is also useful to compute separate structure functions (SF) for the X-ray and optical variations, to compare the variability properties of this sample with previous analyses. We therefore define

$S F_{X}(\tau)=\sqrt{\pi / 2}\left\langle\left|\log F_{X}(t+\tau)-\log F_{X}(t)\right|\right\rangle$,

where $F_{\mathrm{X}}$ is the X-ray flux in the observed $0.2-2 \mathrm{keV}$ band. This is similar to the definition introduced by us (Vagnetti et al. 2011), except that we here omit the subtraction of the contribution due

\footnotetext{
3 Due to a misprint, an incorrect factor $\pi / 2$ was written in Paper I. The correct factor $\sqrt{\pi / 2}$ was used in the computations, however.
}

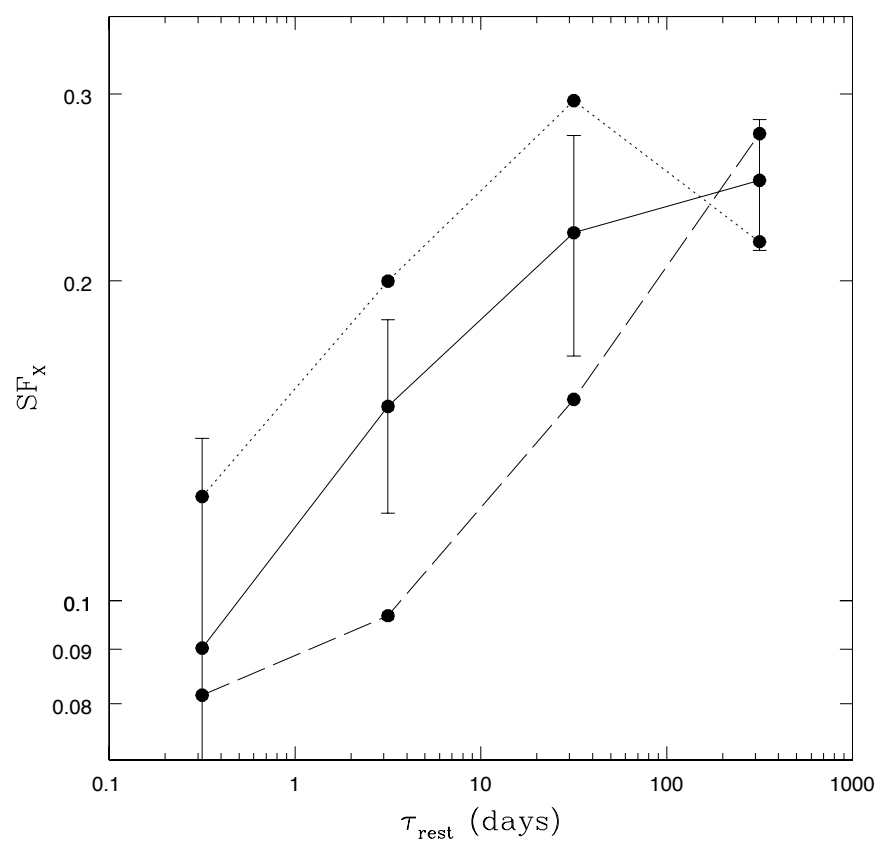

Fig. 8. Binned ensemble structure function of the $\mathrm{X}$-ray flux in the $0.2-2 \mathrm{keV}$ band vs. the rest-frame time lag. Continuous line: whole sample B; dotted line: NLS1; dashed line: BLS1.

to photometric noise, which turns out to be negligible in this case $\left(\sigma_{\mathrm{n}} \sim 0.01\right)$.

The structure function, shown in Fig. 8, represents a variability of $\geq 0.2$ at $\sim 1 \mathrm{yr}$ in the logarithm, or $\sim 60 \%$. This can be compared with the SF obtained by us from the XMM-Newton serendipitous source catalogue (Vagnetti et al. 2011), which has similar levels of variability at all timescales. The observed X-ray band in that case is $0.5-4.5 \mathrm{keV}$, which translates into $\sim 1-11 \mathrm{keV}$ for the higher redshifts of that sample. The luminosities are also different, but the same variability is found for the lower luminosity sources in the Vagnetti et al. (2011) sample, which are comparable to the sources in the present sample. For lowredshift AGNs, most authors use normalised excess variance or fractional variability, and no structure function analyses are available. The energy bands are also usually harder. For example, Markowitz \& Edelson (2004) found a fractional variability $F_{\text {var }}$ between $10 \%$ and $70 \%$ for 55 Seyfert 1 AGNs at months-to-years timescale in the $2-4 \mathrm{keV}$ band. Chitnis et al. (2009) found an average long-term fractional variability $\sim 60 \%$ in the $1.5-3 \mathrm{keV}$ band for $\sim 30$ Seyferts. No direct comparisons are available in the $0.2-2 \mathrm{keV}$ band; energy-dependent analyses (Gierliński \& Done 2006; Arévalo et al. 2008) report a peak of variability around 1-2 keV for a few Seyferts, with decreasing variability both at lower and higher energies, although different behaviours are found for other sources. With these limitations, our SF in Fig. 8 compares reasonably well with other results for similar AGN populations.

\subsection{Optical/UV SF}

Here, we define

$S F_{O}(\tau)=\sqrt{\pi / 2}\langle|m(t+\tau)-m(t)|\rangle$,

where $m$ is the apparent magnitude in any of the UVOT bands. We omitted noise subtraction in this case as well. We stress that optical variabilities measured through Eq. (13) differ from the $\mathrm{X}$-ray variabilities measured through Eq. (12) by the factor 2.5 


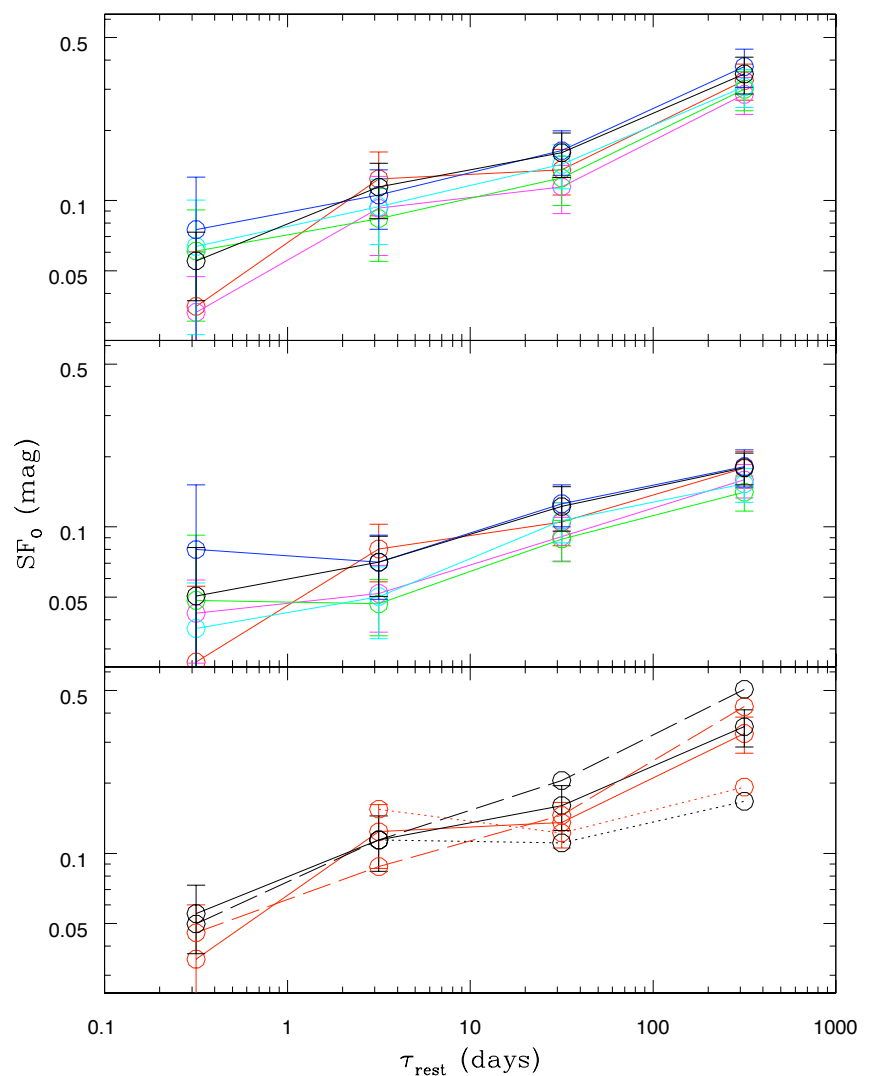

Fig. 9. UV/optical structure function. Upper panel: whole sample B, UVOT filters $U V W 2$ (black), $U V M 2$ (blue), $U V W 1$ (cyan), $U$ (green), $B$ (magenta), $V$ (red). Middle panel: subsample with $L_{\mathrm{UV}} \geq$ $10^{29} \mathrm{erg} \mathrm{s}^{-1} \mathrm{~Hz}^{-1}$, same colour-code. Lower panel: NLS1 (dotted lines), BLS1 (dashed lines), whole sample B (continuous lines). Only the $U V W 2$ filter (black) and the $V$ filter (red) are shown.

introduced by the magnitudes, that are usually adopted in optical studies.

The result is shown in Fig. 9, upper panel, for the six UVOT bands, and represents a variability of $\sim 0.3-0.4$ mag at $\sim 1 \mathrm{yr}$. The values of our SFs can be compared with many other SF analyses, although most of them refer to quasars at higher redshifts. For example, Vanden Berk et al. (2004) found $S F \sim$ 0.2-0.15 in the SDSS gri bands, and Wilhite et al. (2008) $S F \sim 0.3-0.15$ in the ugriz bands, which scan an overall restframe range $\sim 1400-3600 \AA$ at $\langle z\rangle \sim 1.5$. MacLeod et al. (2012) found $S F \lesssim 0.2$ in the rest-frame interval 2000-3000. . For the low-redshift AGNs of the present sample, the above $\lambda$ intervals are well covered by the four harder UVOT bands, $U V W 2$, $U V M 2, U V W 1, U$, where our SFs are $\gtrsim 0.3 \mathrm{mag}$. The variability of our sample is then slightly higher than the variability of the comparison samples. This is also due to the lower luminosity of our sources, however, and to the well-known fact that variability decreases with luminosity (e.g., as $L^{-0.246}$ following Vanden Berk et al. 2004). Therefore, we extracted from our sample B a subsample of sources with $\left\langle L_{\mathrm{UV}}\right\rangle \geq 10^{29} \mathrm{erg} \mathrm{s}^{-1} \mathrm{~Hz}^{-1}$, well-matched with the luminosities of the Vanden Berk et al. (2004) and Wilhite et al. (2008) samples. The SFs for this subsample, shown in the middle panel of Fig. 9, amount to $\lesssim 0.2$ mag at $1 \mathrm{yr}$, similar to the comparison samples.

\subsection{NLS1s}

The presence of several NLS1 AGNs in our sample (28 NLS1 among 61 multi-epoch sources of sample B) gives us the opportunity to measure their variability compared with broad line Seyfert 1 galaxies (BLS1). NLS1 are known to be strongly X-ray variable for timescales $\lesssim 1$ day (e.g. Leighly 1999), and have been suggested to be strongly variable even at longer timescales (Horikawa et al. 2001). We show in Fig. 8 the X-ray SF of NLS1 (dotted line) and BLS1 (dashed line), compared with the overall behaviour of sample B (continuous line). Clearly, NLS1 vary more than the average, and BLS1 less than the average, on timescales shorter than a few months, while there is no such indication for a lag of $\sim 1 \mathrm{yr}$.

We show in the lower panel of Fig. 9 the optical/UV SFs of our NLS1 (dotted lines) and BLS1 (dashed lines), together with the overall sample B (continuous lines), for the extremal UVOT filters $U V W 2$ (black) and $V$ (red). In both filters, NLS1 are less variable than the average, and BLS1 more variable. Our result confirms previous findings of a weak optical variability of NLS1 (e.g. Ai et al. 2010).

\section{Discussion}

This paper is the second of a series aiming to quantify the contribution of X-ray and UV variability to the dispersion of the $\alpha_{\mathrm{ox}}-L_{\mathrm{UV}}$ anti-correlation. It is confirmed that this contribution is significant ( $\sim 40 \%$ of the total variance for this sample), while the artificial $\alpha_{\text {ox }}$ variability, present in many analyses because of the non-simultaneity of X-ray and UV/optical observations, turns out to be less important in the sense that it is surpassed by the intrinsic $\alpha_{\text {ox }}$ variability. Indeed, strong X-ray and/or UV changes occur for individual sources: while these variations could in principle occur with minor changes of the $\mathrm{X}$-ray/UV ratio, the strong $\alpha_{\text {ox }}$ variations (measured by simultaneous X-ray/UV observations) demonstrate that this is not the case.

Stronger variations occur in the X-rays than in UV. While this behaviour could be affected by the presence of many NLS1, which are strongly variable in X-rays, we have shown that the average variability properties of the analysed sample do not suggest a special effect of this factor.

We also discussed the effect of host galaxy dilution on the slope of the $\alpha_{\mathrm{ox}}-L_{\mathrm{UV}}$ anti-correlation. We showed that the effect is significant for a limited number of low-luminosity sources, still producing a significant flattening of the relation. Even when corrected for the dilution effect, the $\alpha_{\mathrm{ox}}-L_{\mathrm{UV}}$ relation remains flatter than those found at high luminosities by Gibson et al. (2008) and by ourselves (Paper I).

Interestingly, in the recent work by Sazonov et al. (2012), the authors evaluated corona luminosities for a sample of 68 Seyfert galaxies through hard X-ray observations by INTEGRAL and accretion disk luminosities through Spitzer observations of the radiation reprocessed by the torus in the mid-infrared, and estimated a disk/corona luminosity ratio approximately constant over two decades in luminosity. While this apparently would contradict the $\alpha_{\mathrm{ox}}-L_{\mathrm{UV}}$ anti-correlation, the authors argued that the $2500 \AA$ luminosity $L_{\mathrm{UV}}$ is a good indicator of the accretion disk luminosity for quasars, but not for lower luminosity AGNs, which are expected to have smaller mass black holes, and hotter accretion disks, with emission peaked in the extreme UV, rather than in the near-UV. This would suggest that $\alpha_{\text {ox }}$ is nearly constant at low luminosities, but this indication is not supported by our findings, Eq. (8), nor by those of Xu (2011).

The variability of $\alpha_{\text {ox }}$, measured by the SFs of Paper I and of the present paper, also gives information on the relation between disk and corona emissions and their variabilities. This relation 
is complex and includes many processes, e.g variable X-ray irradiation driving optical variations through variable heating of the internal parts of the disk on relatively short timescales, and Compton upscattering in the corona by UV/optical photons generated in the accretion disk and variable on longer timescales due to disk instabilities born in the outer parts of the disk and propagating inwards (e.g. Czerny 2006; Arévalo 2006). The SF of $\alpha_{\text {ox }}$, shown in Fig. 7, increases with the time lag up to $\sim 1$ month, while in Paper I another increase at $\sim 1$ year was also present. These findings indicate the substantial contribution of the intermediate-long timescale variations, possibly generated in the outer parts of the accretion disk.

Another interesting aspect concerns the inter-source dispersion, i.e. the residual dispersion of the $\alpha_{\mathrm{ox}}-L_{\mathrm{UV}}$ relation after accounting for the effect of variability. This could be related to the dependence of $\alpha_{\mathrm{ox}}$ on a second physical parameter, in addition to the primary dependence on luminosity. For example, both Lusso et al. (2010) and G10 found evidence of a decrease of $\alpha_{\text {ox }}$ with Eddington ratio. Moreover, Young et al. (2010) found a significant partial anti-correlation with the Eddington ratio when the dependence on $L_{\mathrm{UV}}$ is accounted for; the significance increases if the X-ray energy in the $\alpha_{\mathrm{ox}}$ definition is increased.

Another step in the investigation of the variability of $\alpha_{\mathrm{ox}}$ would be a better temporal sampling of the simultaneous X-ray and optical observations. An appropriate strategy would be a multi-epoch survey of the same field with an X-ray/optical telescope such as XMM-Newton or Swift, and the opportunity is punctually offered by the XMM Deep survey in the Chandra Deep Field South (Comastri et al. 2011). We are preparing an analysis of the individual properties of $\alpha_{\mathrm{ox}}$ variability for the brightest sources, which have simultaneous X-ray/optical multiepoch information (Vagnetti et al, in preparation).

Acknowledgements. We thank Dirk Grupe for help in using his published data, and for suggestions and comments. We thank Maurizio Paolillo, Sara Turriziani, and Guido Risaliti for useful discussions. We thank the referee, who induced us to correcting for the host galaxy contamination. F.V. acknowledges hospitality by the University of Rome "La Sapienza", during his sabbatical leave from the University of Rome "Tor Vergata". This research has made use of data and/or software provided by the High Energy Astrophysics Science Archive Research Center (HEASARC), which is a service of the Astrophysics Science Division at NASA/GSFC and the High Energy Astrophysics Division of the Smithsonian Astrophysical Observatory. This research has made use of the NASA/IPAC Extragalactic Database (NED), which is operated by the Jet Propulsion Laboratory, California Institute of Technology, under contract with the National Aeronautics and Space Administration. No financial resources have been granted to this research by the University of Roma "Tor Vergata".

\section{References}

Ai, Y. L., Yuan, W., Zhou, H. Y., et al. 2010, ApJ, 716, L31

Arévalo, P. 2006, in VI Microquasar Workshop: Microquasars and Beyond, PoS(MQW6)032
Arévalo, P., McHardy, I. M., Markowitz, A., et al. 2008, MNRAS, 387, 279 Bentz, M. C., Peterson, B. M., Netzer, H., Pogge, R. W., \& Vestergaard, M. 2009, ApJ, 697, 160

Brandt, W. N., Laor, A., \& Wills, B. J. 2000, ApJ, 528, 637

Chitnis, V. R., Pendharkar, J. K., Bose, D., et al. 2009, ApJ, 698, 1207

Comastri, A., Ranalli, P., Iwasawa, K., et al. 2011, A\&A, 526, L9

Czerny, B. 2006, in ASP Conf. Ser. 360, eds. C. M. Gaskell, I. M. McHardy, B. M. Peterson, \& S. G. Sergeev, 265

di Clemente, A., Giallongo, E., Natali, G., Trevese, D., \& Vagnetti, F. 1996, ApJ, 463,466

Ganguly, R., Brotherton, M. S., Arav, N., et al. 2007, AJ, 133, 479

Gibson, R. R., Brandt, W. N., \& Schneider, D. P. 2008, ApJ, 685, 773

Gierliński, M., \& Done, C. 2006, MNRAS, 371, L16

Green, P. J., \& Mathur, S. 1996, ApJ, 462, 637

Grupe, D., Thomas, H.-C., \& Beuermann, K. 2001, A\&A, 367, 470

Grupe, D., Komossa, S., Leighly, K. M., \& Page, K. L. 2010, ApJS, 187, 64

Ho, L. C., \& Peng, C. Y. 2001, ApJ, 555, 650

Horikawa, T., Hayashida, K., \& Katayama, H. 2001, in New Century of X-ray Astronomy, eds. H. Inoue, \& H. Kunieda, ASP Conf. Ser., 251, 360

Just, D. W., Brandt, W. N., Shemmer, O., et al. 2007, ApJ, 665, 1004

Kellermann, K. I., Sramek, R., Schmidt, M., Shaffer, D. B., \& Green, R. 1989, AJ, 98, 1195

Kinney, A. L., Bohlin, R. C., Blades, J. C., \& York, D. G. 1991, ApJS, 75, 645 Leighly, K. M. 1999, ApJS, 125, 297

Lusso, E., Comastri, A., Vignali, C., et al. 2010, A\&A, 512, A34

MacLeod, C. L., Ivezić, Ž., Sesar, B., et al. 2012, ApJ, 753, 106

Markowitz, A., \& Edelson, R. 2004, ApJ, 617, 939

Pettini, M., \& Boksenberg, A. 1985, ApJ, 294, L73

Poole, T. S., Breeveld, A. A., Page, M. J., et al. 2008, MNRAS, 383, 627

Press, W. H., Teukolsky, S. A., Vetterling, W. T., \& Flannery, B. P. 1992,

Numerical recipes in FORTRAN. The art of scientific computing

Richards, G. T., Lacy, M., Storrie-Lombardi, L. J., et al. 2006, ApJS, 166, 470

Richards, G. T., Kruczek, N. E., Gallagher, S. C., et al. 2011, AJ, 141, 167

Roming, P. W. A., Kennedy, T. E., Mason, K. O., et al. 2005, Space Sci. Rev., 120,95

Roming, P. W. A., Gronwall, C., vanden Berk, D. E., Page, M. J., \& Boyd, P. T. 2009, Ap\&SS, 320, 203

Sazonov, S., Willner, S. P., Goulding, A. D., et al. 2012, ApJ, 757, 181

Sramek, R. A., \& Weedman, D. W. 1980, ApJ, 238, 435

Steffen, A. T., Strateva, I., Brandt, W. N., et al. 2006, AJ, 131, 2826

Strateva, I. V., Brandt, W. N., Schneider, D. P., Vanden Berk, D. G., \& Vignali, C. 2005, AJ, 130, 387

Sulentic, J. W., Dultzin-Hacyan, D., Marziani, P., et al. 2006, Rev. Mex. Astron. Astrofis., 42, 23

Thomas, H.-C., Beuermann, K., Reinsch, K., et al. 1998, A\&A, 335, 467

Turnshek, D. A., \& Grillmair, C. J. 1986, ApJ, 310, L1

Turnshek, D. A., Monier, E. M., Sirola, C. J., \& Espey, B. R. 1997, ApJ, 476, 40

Vagnetti, F., Turriziani, S., Trevese, D., \& Antonucci, M. 2010, A\&A, 519, A17

Vagnetti, F., Turriziani, S., \& Trevese, D. 2011, A\&A, 536, A84

Vanden Berk, D. E., Wilhite, B. C., Kron, R. G., et al. 2004, ApJ, 601, 692

Voges, W., Aschenbach, B., Boller, T., et al. 1999, A\&A, 349, 389

Watson, M. G., Schröder, A. C., Fyfe, D., et al. 2009, A\&A, 493, 339

Wilhite, B. C., Brunner, R. J., Grier, C. J., Schneider, D. P., \& vanden Berk, D. E. 2008, MNRAS, 383, 1232

Wilkes, B. J., Tananbaum, H., Worrall, D. M., et al. 1994, ApJS, 92, 53

Worrall, D. M., Tananbaum, H., Giommi, P., \& Zamorani, G. 1987, ApJ, 313, 596

Wu, J., Vanden Berk, D., Grupe, D., et al. 2012, ApJS, 201, 10

Xu, Y.-D. 2011, ApJ, 739, 64

Young, M., Elvis, M., \& Risaliti, G. 2010, ApJ, 708, 1388

Zamorani, G., Henry, J. P., Maccacaro, T., et al. 1981, ApJ, 245, 357

Pages 9 to 12 are available in the electronic edition of the journal at http://www . aanda. org 
F. Vagnetti et al.: X-ray/UV ratio of AGNs. II.

Table 1. Sources.

\begin{tabular}{|c|c|c|c|c|c|c|c|c|c|c|}
\hline$N_{\text {sou }}$ & Name & $N_{\text {epo }}$ & Epoch (MJD) & $z$ & $\alpha_{x}$ & $\log L_{X}$ & $\log L_{\mathrm{UV}}$ & $\log L_{\mathrm{G}}$ & $\alpha_{\mathrm{ox}}$ & $f_{\mathrm{RL}}$ \\
\hline$(1)$ & $(2)$ & (3) & (4) & (5) & $(6)$ & (7) & $(8)$ & (9) & $(10)$ & (11) \\
\hline 1 & Mkn_335 & 1 & 54632.1 & 0.026 & 1.31 & 25.61 & 28.85 & 27.68 & -1.24 & 0 \\
\hline \multirow[t]{2}{*}{2} & ESO 242-G008 & 1 & 53964.1 & 0.059 & 0.74 & 25.59 & 28.3 & 27.98 & -1.04 & -1 \\
\hline & & 2 & 54044.6 & & 1.17 & 25.38 & 28.61 & & -1.24 & \\
\hline \multirow[t]{3}{*}{3} & Ton S 180 & 1 & 53990.3 & 0.062 & 1.56 & 25.89 & 29.65 & 27.77 & -1.44 & -1 \\
\hline & & 2 & 54236.4 & & 1.48 & 26.0 & 29.6 & & -1.38 & \\
\hline & & 3 & 54465.4 & & 1.58 & 26.08 & 29.73 & & -1.4 & \\
\hline \multirow[t]{2}{*}{4} & QSO 0056-36 & 1 & 53873.9 & 0.165 & 1.06 & 26.58 & 30.18 & - & -1.38 & -1 \\
\hline & & 2 & 53878.6 & & 1.13 & 26.46 & 30.15 & & -1.42 & \\
\hline \multirow[t]{4}{*}{5} & RX J0100.4-5113 & 1 & 54454.5 & 0.062 & 1.08 & 25.54 & 29.12 & 28.03 & -1.37 & -1 \\
\hline & & 2 & 54722.1 & & 1.19 & 25.43 & 29.11 & & -1.41 & \\
\hline & & 3 & 54776.5 & & 1.36 & 25.58 & 29.18 & & -1.38 & \\
\hline & & 4 & 54794.2 & & 1.36 & 25.71 & 29.21 & & -1.34 & \\
\hline \multirow[t]{4}{*}{6} & RX J0105.6-1416 & 1 & 54475.7 & 0.07 & 0.92 & 25.92 & 29.14 & 28.14 & -1.23 & -1 \\
\hline & & 2 & 54599.9 & & 0.99 & 25.96 & 29.26 & & -1.27 & \\
\hline & & 3 & 54633.4 & & 1.14 & 25.98 & 29.27 & & -1.26 & \\
\hline & & 4 & 54643.5 & & 1.02 & 25.97 & 29.22 & & -1.25 & \\
\hline \multirow[t]{3}{*}{7} & RX J0117.5-3826 & 1 & 54646.7 & 0.225 & 1.73 & 26.1 & 29.58 & 28.78 & -1.34 & -1 \\
\hline & & 2 & 54949.5 & & 2.14 & 26.03 & 29.51 & & -1.33 & \\
\hline & & 3 & 55145.5 & & 2.09 & 25.79 & 29.49 & & -1.42 & \\
\hline 8 & MS 0117-28 & 1 & 54044.3 & 0.349 & 1.6 & 26.37 & 30.53 & - & -1.6 & -1 \\
\hline \multirow[t]{4}{*}{9} & RX J0128.1-1848 & 1 & 54275.5 & 0.046 & 0.92 & 25.76 & 28.89 & 28.23 & -1.2 & -1 \\
\hline & & 2 & 54501.5 & & 0.89 & 25.69 & 28.83 & & -1.21 & \\
\hline & & 3 & 54502.5 & & 1.1 & 25.71 & 28.84 & & -1.2 & \\
\hline & & 4 & 54503.5 & & 1.01 & 25.74 & 28.84 & & -1.19 & \\
\hline 10 & RX J0134.2-4258 & 1 & 54433.5 & 0.237 & 1.29 & 26.26 & 30.25 & - & -1.53 & 1 \\
\hline 11 & RX J0136.9-3510 & 1 & 53767.0 & 0.289 & 1.87 & 26.54 & 29.79 & 28.29 & -1.25 & -1 \\
\hline \multirow[t]{6}{*}{12} & RX J0148.3-2758 & 1 & 54037.3 & 0.121 & 1.75 & 26.33 & 29.71 & 28.68 & -1.3 & -1 \\
\hline & & 2 & 54232.2 & & 1.82 & 26.12 & 29.72 & & -1.38 & \\
\hline & & 3 & 54429.6 & & 1.73 & 26.35 & 29.68 & & -1.28 & \\
\hline & & 4 & 54593.2 & & 1.81 & 26.25 & 29.67 & & -1.31 & \\
\hline & & 5 & 54633.9 & & 1.67 & 26.34 & 29.7 & & -1.29 & \\
\hline & & 6 & 54644.3 & & 1.57 & 26.13 & 29.65 & & -1.35 & \\
\hline \multirow[t]{3}{*}{13} & RX J0152.4-2319 & 1 & 54467.7 & 0.113 & 1.25 & 25.99 & 29.69 & 28.33 & -1.42 & 0 \\
\hline & & 2 & 54508.9 & & 1.24 & 26.14 & 29.7 & & -1.37 & \\
\hline & & 3 & 54514.6 & & 1.23 & 26.2 & 29.71 & & -1.35 & \\
\hline \multirow[t]{2}{*}{14} & Mkn 1044 & 1 & 54306.6 & 0.017 & 1.46 & 25.01 & 28.33 & 27.35 & -1.28 & 1 \\
\hline & & 2 & 54313.5 & & 1.47 & 24.68 & 28.34 & & -1.41 & \\
\hline 15 & Mkn 1048 & 1 & 54304.5 & 0.042 & 0.61 & 25.42 & 29.13 & 27.87 & -1.42 & 0 \\
\hline & & 2 & 54451.8 & & 1.02 & 25.94 & 29.2 & & -1.25 & \\
\hline & & 3 & 54529.4 & & 0.71 & 25.82 & 29.18 & & -1.29 & \\
\hline & & 4 & 54624.3 & & 0.78 & 25.84 & 29.18 & & -1.28 & \\
\hline 16 & RX J0311.3-2046 & 1 & 54916.7 & 0.07 & 0.83 & 25.93 & 28.99 & 28.0 & -1.18 & -1 \\
\hline & & 2 & 54982.6 & & 0.87 & 25.85 & 29.02 & & -1.22 & \\
\hline 17 & RX J0319.8-2627 & 1 & 54166.5 & 0.076 & 0.82 & 25.6 & 28.71 & 28.27 & -1.2 & -1 \\
\hline & & 2 & 54169.2 & & 0.94 & 25.49 & 28.63 & & -1.21 & \\
\hline & & 3 & 54174.5 & & 0.87 & 25.54 & 28.69 & & -1.21 & \\
\hline & & 4 & 54175.1 & & 0.7 & 25.51 & 28.68 & & -1.22 & \\
\hline & & 5 & 54546.8 & & 1.08 & 25.62 & 28.98 & & -1.29 & \\
\hline & & 6 & 54559.5 & & 1.05 & 25.83 & 29.06 & & -1.24 & \\
\hline & & 7 & 54798.3 & & 0.55 & 25.36 & 28.54 & & -1.22 & \\
\hline & & 8 & 54911.5 & & 0.98 & 25.59 & 28.73 & & -1.2 & \\
\hline 18 & RX J0323.2-4911 & 1 & 54441.9 & 0.071 & 1.33 & 25.01 & 27.77 & 28.23 & -1.06 & -1 \\
\hline & & 2 & 54449.4 & & 1.08 & 25.29 & 27.73 & & -0.94 & \\
\hline 19 & ESO 301-G13 & 1 & 54779.6 & 0.059 & 1.35 & 25.62 & 28.96 & 28.01 & -1.28 & -1 \\
\hline & & 2 & 54810.6 & & 1.19 & 25.57 & 28.81 & & -1.24 & \\
\hline & & 3 & 55122.4 & & 1.1 & 25.75 & 28.93 & & -1.22 & \\
\hline & & 4 & 55194.8 & & 1.31 & 25.6 & 28.94 & & -1.28 & \\
\hline 20 & VCV 0331-37 & 1 & 54650.7 & 0.064 & 1.04 & 25.53 & 28.81 & 27.62 & -1.26 & -1 \\
\hline & & 2 & 54766.1 & & 1.07 & 25.5 & 28.8 & & -1.27 & \\
\hline & & 3 & 54913.6 & & 1.24 & 25.51 & 28.87 & & -1.29 & \\
\hline & & 4 & 54915.3 & & 1.19 & 25.55 & 28.88 & & -1.28 & \\
\hline 21 & RX J0349.1-4711 & 1 & 53974.5 & 0.299 & 1.62 & 26.39 & 29.98 & 28.65 & -1.38 & -1 \\
\hline & & 2 & 54185.9 & & 1.35 & 26.53 & 29.98 & & -1.33 & \\
\hline 22 & Fairall 1116 & 1 & 53752.1 & 0.059 & 1.05 & 25.94 & 29.17 & 28.13 & -1.24 & -1 \\
\hline & & 2 & 53833.6 & & 1.33 & 25.74 & 29.26 & & -1.35 & \\
\hline
\end{tabular}


A\&A 550, A71 (2013)

Table 1. continued.

\begin{tabular}{|c|c|c|c|c|c|c|c|c|c|c|}
\hline$N_{\text {sou }}$ & Name & $N_{\text {epo }}$ & Epoch (MJD) & $z$ & $\alpha_{x}$ & $\log L_{X}$ & $\log L_{\mathrm{UV}}$ & $\log L_{\mathrm{G}}$ & $\alpha_{\mathrm{ox}}$ & $f_{\mathrm{RL}}$ \\
\hline & & 3 & 53857.5 & & 1.35 & 25.54 & 29.28 & & -1.43 & \\
\hline \multirow[t]{2}{*}{23} & Fairall 1119 & 1 & 54751.9 & 0.055 & 0.72 & 25.39 & 27.65 & 28.07 & -0.87 & -1 \\
\hline & & 2 & 54765.2 & & 0.6 & 25.3 & 27.69 & & -0.92 & \\
\hline \multirow[t]{2}{*}{24} & RX J0412.7-4712 & 1 & 54459.3 & 0.132 & 1.02 & 26.42 & 29.68 & 28.57 & -1.25 & -1 \\
\hline & & 2 & 54467.3 & & 1.09 & 26.61 & 29.72 & & -1.2 & \\
\hline \multirow[t]{2}{*}{25} & 1H 0419-577 & 1 & 54761.4 & 0.104 & 1.22 & 26.81 & 29.92 & 28.9 & -1.19 & 0 \\
\hline & & 2 & 54782.6 & & 1.07 & 26.62 & 29.9 & & -1.26 & \\
\hline \multirow[t]{2}{*}{26} & Fairall 303 & 1 & 54768.2 & 0.04 & 1.32 & 25.23 & 28.37 & 27.47 & -1.2 & -1 \\
\hline & & 2 & 54787.7 & & 1.17 & 25.08 & 28.31 & & -1.24 & \\
\hline \multirow[t]{2}{*}{27} & RX J0437.4-4711 & 1 & 54441.6 & 0.052 & 1.2 & 25.67 & 29.0 & 28.15 & -1.28 & -1 \\
\hline & & 2 & 54452.1 & & 1.17 & 25.83 & 29.0 & & -1.22 & \\
\hline \multirow[t]{4}{*}{28} & RX J0439.6-5311 & 1 & 53741.3 & 0.243 & 2.16 & 26.67 & 29.7 & - & -1.17 & -1 \\
\hline & & 2 & 53838.3 & & 2.12 & 26.64 & 29.7 & & -1.17 & \\
\hline & & 3 & 53840.5 & & 2.05 & 26.79 & 29.7 & & -1.11 & \\
\hline & & 4 & 53873.7 & & 2.07 & 26.73 & 29.69 & & -1.14 & \\
\hline \multirow[t]{3}{*}{29} & RX J0859.0+4866 & 1 & 54020.5 & 0.083 & 0.91 & 25.94 & 29.23 & 28.4 & -1.26 & -1 \\
\hline & & 2 & 54225.1 & & 0.98 & 25.92 & 29.26 & & -1.28 & \\
\hline & & 3 & 54239.3 & & 1.14 & 25.91 & 29.32 & & -1.31 & \\
\hline \multirow[t]{3}{*}{30} & RX J0902.5-0700 & 1 & 54269.1 & 0.089 & 1.23 & 25.16 & 28.63 & 27.68 & -1.33 & -1 \\
\hline & & 2 & 54461.6 & & 1.24 & 25.34 & 28.77 & & -1.32 & \\
\hline & & 3 & 54467.5 & & 1.61 & 25.31 & 28.78 & & -1.33 & \\
\hline \multirow[t]{2}{*}{31} & Mkn 110 & 1 & 55202.8 & 0.035 & 0.98 & 26.13 & 29.07 & 26.4 & -1.13 & 0 \\
\hline & & 2 & 55208.7 & & 1.02 & 26.12 & 29.09 & & -1.14 & \\
\hline 33 & RX J1005.7+4332 & 1 & 53789.6 & 0.178 & 1.8 & 25.99 & 29.84 & - & -1.48 & 0 \\
\hline \multirow[t]{2}{*}{34} & RX J1007.1+2203 & 1 & 54281.8 & 0.083 & 1.5 & 25.4 & 28.71 & 27.76 & -1.27 & -1 \\
\hline & & 2 & 54647.4 & & 1.53 & 25.11 & 28.72 & & -1.39 & \\
\hline 35 & CBS 126 & 1 & 53899.2 & 0.079 & 1.4 & 25.6 & 29.3 & 28.21 & -1.42 & -1 \\
\hline & & 2 & 54132.7 & & 1.39 & 25.78 & 29.37 & & -1.38 & 1 \\
\hline 36 & Mkn 141 & 1 & 54023.5 & 0.042 & 0.76 & 25.08 & 28.17 & 28.05 & -1.19 & 0 \\
\hline & & 2 & 54185.9 & & 0.36 & 24.52 & 28.26 & & -1.44 & \\
\hline 37 & Mkn 142 & 1 & 54428.7 & 0.045 & 1.38 & 25.17 & 28.63 & 27.61 & -1.33 & 0 \\
\hline & & 2 & 54479.9 & & 1.72 & 25.41 & 28.74 & & -1.28 & \\
\hline 38 & RX J1117.1+6522 & 1 & 54054.5 & 0.147 & 1.93 & 25.78 & 29.36 & 28.87 & -1.37 & -1 \\
\hline & & 2 & 54182.8 & & 1.05 & 25.26 & 29.25 & & -1.53 & \\
\hline & & 3 & 54195.6 & & 2.09 & 25.98 & 29.39 & & -1.31 & \\
\hline & & 4 & 54220.7 & & 1.91 & 25.4 & 29.36 & & -1.52 & \\
\hline 39 & Ton 1388 & 1 & 54289.6 & 0.177 & 1.26 & 26.71 & 30.64 & - & -1.51 & 0 \\
\hline & & 2 & 54940.4 & & 1.43 & 26.7 & 30.67 & & -1.53 & \\
\hline 40 & EXO 1128+6908 & 1 & 54878.8 & 0.045 & 1.24 & 25.58 & 28.6 & 27.93 & -1.19 & -1 \\
\hline 41 & B2 $1128+31$ & 1 & 54753.7 & 0.289 & 1.05 & 26.89 & 30.42 & - & -1.35 & 1 \\
\hline & & 2 & 54879.7 & & 0.99 & 26.96 & 30.35 & & -1.3 & \\
\hline & & 3 & 55163.7 & & 1.14 & 26.82 & 30.32 & & -1.34 & \\
\hline 42 & SBS $1136+579$ & 1 & 54182.5 & 0.116 & 0.98 & 25.83 & 29.14 & 28.26 & -1.27 & -1 \\
\hline & & 2 & 54936.6 & & 1.32 & 25.35 & 28.71 & & -1.29 & \\
\hline 43 & CASG 855 & 1 & 54883.7 & 0.04 & 0.83 & 25.0 & 27.21 & 27.76 & -0.85 & -1 \\
\hline 44 & NGC 4051 & 1 & 54876.1 & 0.0020 & 1.59 & 23.72 & 26.35 & 26.08 & -1.01 & 0 \\
\hline 45 & GQ Comae & 1 & 54762.5 & 0.165 & 1.1 & 26.67 & 29.82 & - & -1.21 & -1 \\
\hline & & 2 & 54763.5 & & 1.01 & 26.7 & 29.8 & & -1.19 & \\
\hline 46 & RX J1209.8+3217 & 1 & 54269.0 & 0.145 & 1.86 & 25.26 & 29.08 & 28.26 & -1.47 & 0 \\
\hline & & 2 & 54760.5 & & 3.07 & 24.6 & 29.2 & & -1.76 & \\
\hline 47 & PG $1211+143$ & 1 & 54192.9 & 0.082 & 1.89 & 25.28 & 29.81 & 28.74 & -1.74 & 0 \\
\hline 48 & Mkn 766 & 1 & 54090.9 & 0.013 & 1.05 & 24.73 & 26.33 & 27.45 & -0.61 & 1 \\
\hline 49 & $3 \mathrm{C} 273$ & 1 & 54863.9 & 0.158 & 0.72 & 27.82 & 31.19 & - & -1.29 & 0 \\
\hline & & 2 & 54919.5 & & 0.74 & 27.79 & 31.19 & & -1.3 & \\
\hline 50 & RX J1231.6+7044 & 1 & 54236.0 & 0.208 & 0.6 & 26.88 & 29.96 & 28.54 & -1.18 & -1 \\
\hline & & 2 & 54282.7 & & 0.82 & 26.86 & 29.97 & & -1.19 & \\
\hline & & 3 & 54293.6 & & 0.85 & 26.83 & 29.94 & & -1.19 & \\
\hline & & 4 & 54294.2 & & 0.87 & 26.82 & 29.94 & & -1.2 & \\
\hline 51 & MCG+08-23-006 & 1 & 54190.0 & 0.03 & 0.82 & 24.52 & 27.31 & 27.62 & -1.07 & 0 \\
\hline 52 & NGC 4593 & 1 & 54693.8 & 0.0090 & 0.69 & 24.64 & 27.22 & 27.25 & -0.99 & 0 \\
\hline 53 & RX J1304.2+0205 & 1 & 54444.5 & 0.229 & 1.97 & 25.97 & 29.64 & 27.84 & -1.41 & -1 \\
\hline & & 2 & 54681.5 & & 2.17 & 26.16 & 29.7 & & -1.36 & \\
\hline 54 & PG $1307+085$ & 1 & 54696.0 & 0.155 & 1.16 & 26.44 & 30.09 & - & -1.4 & 0 \\
\hline & & 2 & 54697.4 & & 1.26 & 26.44 & 30.09 & & -1.4 & \\
\hline 55 & RX J1319.9+5235 & 1 & 54028.3 & 0.092 & 1.87 & 25.37 & 28.07 & 27.81 & -1.04 & -1 \\
\hline & & 2 & 54187.9 & & 1.86 & 25.45 & 28.12 & & -1.03 & \\
\hline & & 3 & 54195.6 & & 1.64 & 25.65 & 28.07 & & -0.93 & \\
\hline
\end{tabular}


F. Vagnetti et al.: X-ray/UV ratio of AGNs. II.

Table 1. continued.

\begin{tabular}{|c|c|c|c|c|c|c|c|c|c|c|}
\hline$N_{\text {sou }}$ & Name & $N_{\text {epo }}$ & Epoch (MJD) & $z$ & $\alpha_{x}$ & $\log L_{X}$ & $\log L_{\mathrm{UV}}$ & $\log L_{\mathrm{G}}$ & $\alpha_{\mathrm{ox}}$ & $f_{\mathrm{RL}}$ \\
\hline 57 & Ton 730 & 1 & 55057.2 & 0.087 & 1.41 & 25.83 & 29.3 & 27.02 & -1.33 & -1 \\
\hline \multirow[t]{5}{*}{58} & RX J1355.2+5612 & 1 & 54233.5 & 0.122 & 1.77 & 25.77 & 29.14 & 28.41 & -1.3 & 0 \\
\hline & & 2 & 54241.9 & & 1.66 & 26.12 & 29.11 & & -1.15 & \\
\hline & & 3 & 54272.4 & & 1.61 & 25.69 & 29.1 & & -1.31 & \\
\hline & & 4 & 54277.8 & & 1.79 & 25.84 & 29.12 & & -1.26 & \\
\hline & & 5 & 54279.5 & & 2.09 & 25.7 & 29.09 & & -1.3 & \\
\hline \multirow[t]{3}{*}{59} & PG $1402+261$ & 1 & 53923.3 & 0.164 & 1.35 & 26.35 & 30.19 & - & -1.48 & -1 \\
\hline & & 2 & 53948.4 & & 1.46 & 26.35 & 30.2 & & -1.48 & \\
\hline & & 3 & 53960.9 & & 1.39 & 26.39 & 30.22 & & -1.47 & \\
\hline \multirow[t]{2}{*}{60} & RX J1413.6+7029 & 1 & 54600.8 & 0.107 & 0.64 & 25.69 & 28.14 & 28.24 & -0.94 & -1 \\
\hline & & 2 & 54905.7 & & 0.97 & 26.02 & 28.74 & & -1.04 & \\
\hline 61 & NGC 5548 & 1 & 54270.4 & 0.017 & 0.41 & 24.65 & 27.67 & 27.59 & -1.16 & 0 \\
\hline 62 & Mkn 813 & 1 & 54110.3 & 0.111 & 1.0 & 26.38 & 30.03 & 27.05 & -1.4 & -1 \\
\hline \multirow[t]{2}{*}{63} & Mkn 684 & 1 & 53879.5 & 0.046 & 1.42 & 25.27 & 29.07 & 28.1 & -1.46 & 1 \\
\hline & & 2 & 53880.5 & & 1.24 & 25.42 & 29.1 & & -1.41 & \\
\hline \multirow[t]{2}{*}{64} & Mkn 478 & 1 & 53976.8 & 0.077 & 1.41 & 25.52 & 29.71 & 28.35 & -1.61 & 0 \\
\hline & & 2 & 54017.0 & & 1.35 & 25.76 & 29.71 & & -1.51 & \\
\hline \multirow[t]{4}{*}{66} & Mkn 841 & 1 & 54101.5 & 0.036 & 0.87 & 25.54 & 28.97 & 27.59 & -1.32 & 0 \\
\hline & & 2 & 54133.3 & & 0.96 & 25.59 & 29.0 & & -1.31 & \\
\hline & & 3 & 54134.4 & & 0.91 & 25.49 & 29.0 & & -1.35 & \\
\hline & & 4 & 54621.3 & & 1.05 & 25.59 & 29.06 & & -1.33 & \\
\hline \multirow[t]{3}{*}{67} & Mkn 493 & 1 & 53679.7 & 0.032 & 1.29 & 25.01 & 28.57 & 27.65 & -1.37 & 0 \\
\hline & & 2 & 53682.6 & & 1.32 & 25.12 & 28.4 & & -1.26 & \\
\hline & & 3 & 54622.5 & & 1.12 & 25.0 & 28.43 & & -1.32 & \\
\hline \multirow[t]{2}{*}{68} & Mkn 876 & 1 & 53885.7 & 0.129 & 0.9 & 26.42 & 30.26 & 28.4 & -1.47 & 0 \\
\hline & & 2 & 53905.5 & & 1.01 & 26.35 & 30.28 & & -1.51 & \\
\hline \multirow[t]{2}{*}{70} & KUG $1618+410$ & 1 & 54475.9 & 0.038 & 0.9 & 24.63 & 27.75 & 27.63 & -1.2 & -1 \\
\hline & & 2 & 54479.7 & & 0.98 & 24.67 & 27.61 & & -1.13 & \\
\hline \multirow[t]{2}{*}{71} & PG $1626+554$ & 1 & 54606.4 & 0.133 & 1.11 & 26.33 & 29.82 & - & -1.34 & -1 \\
\hline & & 2 & 54618.9 & & 1.32 & 26.27 & 29.84 & & -1.37 & \\
\hline 72 & EXO $1627+40$ & 1 & 54277.3 & 0.272 & 1.15 & 26.56 & 29.55 & 27.9 & -1.15 & 1 \\
\hline & & 2 & 54473.3 & & 1.06 & 26.61 & 29.53 & & -1.12 & \\
\hline & & 3 & 54477.1 & & 1.41 & 26.4 & 29.55 & & -1.21 & \\
\hline 73 & RX J1702.5+3247 & 1 & 53978.5 & 0.164 & 1.73 & 26.22 & 29.94 & 28.94 & -1.43 & 0 \\
\hline & & 2 & 54019.5 & & 1.79 & 26.37 & 29.95 & & -1.37 & \\
\hline & & 3 & 54119.5 & & 1.16 & 26.08 & 29.94 & & -1.48 & \\
\hline & & 4 & 54123.4 & & 1.67 & 26.19 & 29.94 & & -1.44 & \\
\hline 74 & II Zw 136 & 1 & 54665.5 & 0.065 & 1.32 & 26.07 & 29.65 & 28.31 & -1.38 & 0 \\
\hline & & 2 & 54666.6 & & 1.38 & 26.09 & 29.65 & & -1.37 & \\
\hline & & 3 & 54684.2 & & 1.49 & 25.82 & 29.57 & & -1.44 & \\
\hline & & 4 & 54790.5 & & 1.24 & 25.92 & 29.59 & & -1.41 & \\
\hline 76 & RX J2216.8-4451 & 1 & 54659.4 & 0.136 & 1.5 & 26.09 & 29.8 & - & -1.43 & -1 \\
\hline & & 2 & 54661.6 & & 1.69 & 26.13 & 29.8 & & -1.41 & \\
\hline & & 3 & 54671.3 & & 1.66 & 26.4 & 29.81 & & -1.31 & \\
\hline & & 4 & 54673.3 & & 1.48 & 26.41 & 29.83 & & -1.31 & \\
\hline 77 & RX J2217.9-5941 & 1 & 54042.1 & 0.16 & 2.01 & 25.45 & 29.44 & 28.3 & -1.53 & 0 \\
\hline & & 2 & 54220.1 & & 2.45 & 25.21 & 29.44 & & -1.62 & \\
\hline 78 & RX J2242.6-3845 & 1 & 54665.9 & 0.221 & 1.68 & 26.04 & 29.65 & 28.18 & -1.38 & -1 \\
\hline & & 2 & 54668.9 & & 1.6 & 26.36 & 29.68 & & -1.27 & \\
\hline 79 & RX J2245.3-4652 & 1 & 53898.5 & 0.201 & 1.16 & 26.28 & 30.23 & 29.28 & -1.52 & -1 \\
\hline & & 2 & 53901.8 & & 1.27 & 26.16 & 30.24 & & -1.56 & \\
\hline 81 & MS 2254-36 & 1 & 54274.9 & 0.039 & 1.19 & 25.22 & 28.3 & 27.73 & -1.18 & -1 \\
\hline & & 2 & 54316.6 & & 1.14 & 25.24 & 28.34 & & -1.19 & \\
\hline & & 3 & 54441.5 & & 1.14 & 25.08 & 28.27 & & -1.22 & \\
\hline & & 4 & 54445.3 & & 1.18 & 25.19 & 28.31 & & -1.2 & \\
\hline 82 & RX J2258.7-2609 & 1 & 54671.1 & 0.076 & 0.71 & 25.85 & 28.76 & 28.01 & -1.12 & -1 \\
\hline & & 2 & 54680.8 & & 0.98 & 25.88 & 28.8 & & -1.12 & \\
\hline & & 3 & 54725.8 & & 0.88 & 25.66 & 28.89 & & -1.24 & \\
\hline & & 4 & 54794.0 & & 0.91 & 25.7 & 28.74 & & -1.17 & \\
\hline 83 & RX J2301.6-5913 & 1 & 54673.3 & 0.149 & 0.82 & 26.48 & 29.31 & 28.34 & -1.09 & 1 \\
\hline & & 2 & 54681.2 & & 0.84 & 26.5 & 29.33 & & -1.09 & \\
\hline & & 3 & 55032.7 & & 0.77 & 26.62 & 29.3 & & -1.03 & \\
\hline & & 4 & 55098.8 & & 0.86 & 26.57 & 29.42 & & -1.09 & \\
\hline & & 5 & 55102.8 & & 0.83 & 26.61 & 29.42 & & -1.08 & \\
\hline 84 & RX J2301.8-5508 & 1 & 53700.6 & 0.14 & 1.49 & 25.8 & 29.65 & 28.98 & -1.48 & -1 \\
\hline & & 2 & 53712.2 & & 1.38 & 25.93 & 29.67 & & -1.44 & \\
\hline 85 & RX J2304.6-3501 & 1 & 53997.0 & 0.042 & 1.06 & 24.72 & 27.8 & 27.49 & -1.18 & -1 \\
\hline
\end{tabular}

A71, page 11 of 12 
A\&A 550, A71 (2013)

Table 1. continued.

\begin{tabular}{|c|c|c|c|c|c|c|c|c|c|c|}
\hline$N_{\text {sou }}$ & Name & $N_{\text {epo }}$ & Epoch (MJD) & $z$ & $\alpha_{x}$ & $\log L_{X}$ & $\log L_{\mathrm{UV}}$ & $\log L_{\mathrm{G}}$ & $\alpha_{\mathrm{ox}}$ & $f_{\mathrm{RL}}$ \\
\hline \multirow[t]{2}{*}{86} & RX J2312.5-3404 & 1 & 54320.5 & 0.202 & 0.67 & 26.23 & 29.76 & 28.64 & -1.35 & 1 \\
\hline & & 2 & 54457.7 & & 0.77 & 26.42 & 29.72 & & -1.27 & \\
\hline 87 & RX J2317.8-4422 & 1 & 53843.5 & 0.132 & 2.58 & 25.2 & 29.14 & 28.3 & -1.51 & -1 \\
\hline \multirow[t]{3}{*}{88} & RX J2325.2-3236 & 1 & 54002.1 & 0.216 & 1.08 & 26.68 & 29.97 & - & -1.26 & 1 \\
\hline & & 2 & 54045.8 & & 1.02 & 26.64 & 29.99 & & -1.28 & \\
\hline & & 3 & 54708.0 & & 1.21 & 26.6 & 29.92 & & -1.27 & \\
\hline 89 & IRAS 23226-3843 & 1 & 54676.3 & 0.036 & 0.6 & 25.35 & 28.18 & 28.15 & -1.08 & 0 \\
\hline \multirow[t]{5}{*}{90} & MS 23409-1511 & 1 & 54430.4 & 0.137 & 1.79 & 26.1 & 29.71 & 28.61 & -1.39 & -1 \\
\hline & & 2 & 54481.5 & & 1.77 & 26.15 & 29.75 & & -1.38 & \\
\hline & & 3 & 54484.8 & & 1.87 & 26.13 & 29.72 & & -1.38 & \\
\hline & & 4 & 54485.9 & & 1.78 & 26.09 & 29.74 & & -1.4 & \\
\hline & & 5 & 54621.6 & & 1.91 & 25.86 & 29.74 & & -1.49 & \\
\hline \multirow[t]{3}{*}{91} & RX J2349.4-3126 & 1 & 54231.9 & 0.135 & 1.03 & 25.87 & 28.84 & 28.6 & -1.14 & -1 \\
\hline & & 2 & 54792.8 & & 1.03 & 25.88 & 28.77 & & -1.11 & \\
\hline & & 3 & 55098.5 & & 1.03 & 25.88 & 28.66 & & -1.07 & \\
\hline \multirow[t]{2}{*}{92} & AM 2354-304 & 1 & 54850.5 & 0.033 & 1.16 & 25.01 & 28.42 & 27.79 & -1.31 & -1 \\
\hline & & 2 & 54994.8 & & 1.21 & 24.9 & 28.42 & & -1.35 & \\
\hline
\end{tabular}

